\title{
Machine Learning Based Cloud loT Platform for Intelligent Tourism Information Services
}

Fangfei Bi ( $\square$ 20150610@xpu.edu.cn )

Xi'an University of Architecture and Technology

Haotian Liu

JIKAN Research Institute of Engineering Investigations and Design

Research

Keywords: Smart tourism, Internet of Things (IoT), Machine learning, Hybrid cloud

Posted Date: February 2nd, 2022

DOI: https://doi.org/10.21203/rs.3.rs-1220688/v1

License: (9) This work is licensed under a Creative Commons Attribution 4.0 International License. Read Full License 


\title{
Machine Learning Based Cloud IoT Platform for Intelligent Tourism Information Services
}

\author{
Fangfei Bi ${ }^{1, a^{*}}$, Haotian Liu ${ }^{2, b}$ \\ ${ }^{1} X i$ 'an Polytechnic University; Xi'an University of Architecture and Technology; Xi'an 710055, Shaanxi, \\ China \\ ${ }^{2}$ China JIKAN Research Institute of Engineering Investigations and Design, Co. Ltd, Xi'an 710043, Shaanxi, \\ China \\ aEmail:20150610@xpu.edu.cn \\ ${ }^{b}$ Email: 18629046534@163.com \\ ${ }^{*}$ Corresponding Author
}

\begin{abstract}
Because of the continuous development of the tourism industry, the application of the Internet of Things technology to tourism projects is very promising in the future. Smart tourism takes use of the Internet of Things to improve information exchange. Therefore, in order to meet the needs of the next generation of tourists in the future, the Internet of Things technology will become an indispensable part. As a consequence, based on visitor selection behavior, we offer a hybrid intelligent categorization approach. This method of categorization might help tourists decide whether or not to visit a tourist attraction. Cross-validation testing and performance assessment results indicate the method's effectiveness.
\end{abstract}

Keywords: Smart tourism; Internet of Things (IoT); Machine learning; Hybrid cloud

\section{Introduction}

The Internet of Things (IoT) is an indispensable part of the future Internet ecosystem. In the future, the Internet of Things technology has a very important role and significance for healthcare and e-tourism [1]. In the future, the rapid development of business will be a new field of services that takes into account the background of location and geographic factors. These services are necessary for the development of IoT scenarios, smart environments, and active solutions. In these fields, smart tourism will become one of the most interesting applications [2]. In many areas, the main obstacles to tourism are numerous social issues, such as security, fraud, and lack of resource information. But smart tourism design can help tourists collect and sort out all the surrounding information and data and pass it back to tourists, turning it into valuable data and individual information[3]. The Internet of Things is being used in smart tourism, as seen in Figure 1. Our system also aims to create a tourism industry that can appeal to a wide variety of clients, as well as provide users with cutting-edge entertainment, communication, and information services and applications via a new business model. We have developed a novel travel information search method because of the need of cloud services [4]. By combining smart phones, GPS, Google Maps, and augmented reality, we will provide mobile customers with a travel mode that they never felt before 5]. Today, 5G is the most sophisticated technology on the globe, and its applications are quickly becoming commonplace. The future direction and development potential of 5G smart tourism are highly promising. 5G technology encourages the intelligent upgrade of tourist sites by integrating AR, AI, social sharing, and other apps thanks to its super-speed, high-bandwidth, and low-latency characteristics. Promoting the creation of 5G smart tourism applications is an important step toward promoting tourism across the country and supporting the smart development of attractive regions, and it will be definitely beneficial to attracting tourists.

Tourism is one of the most profitable economic activities on the globe, and it is an important part of the economic growth of many countries [6]. Some countries around the world depend on tourism 
to promote economic development. Indeed, tourism has the potential to greatly accelerate the growth of a number of economic sectors, including local economy revival and job creation [7]. It has the potential to play an important role in promoting international collaboration and understanding.

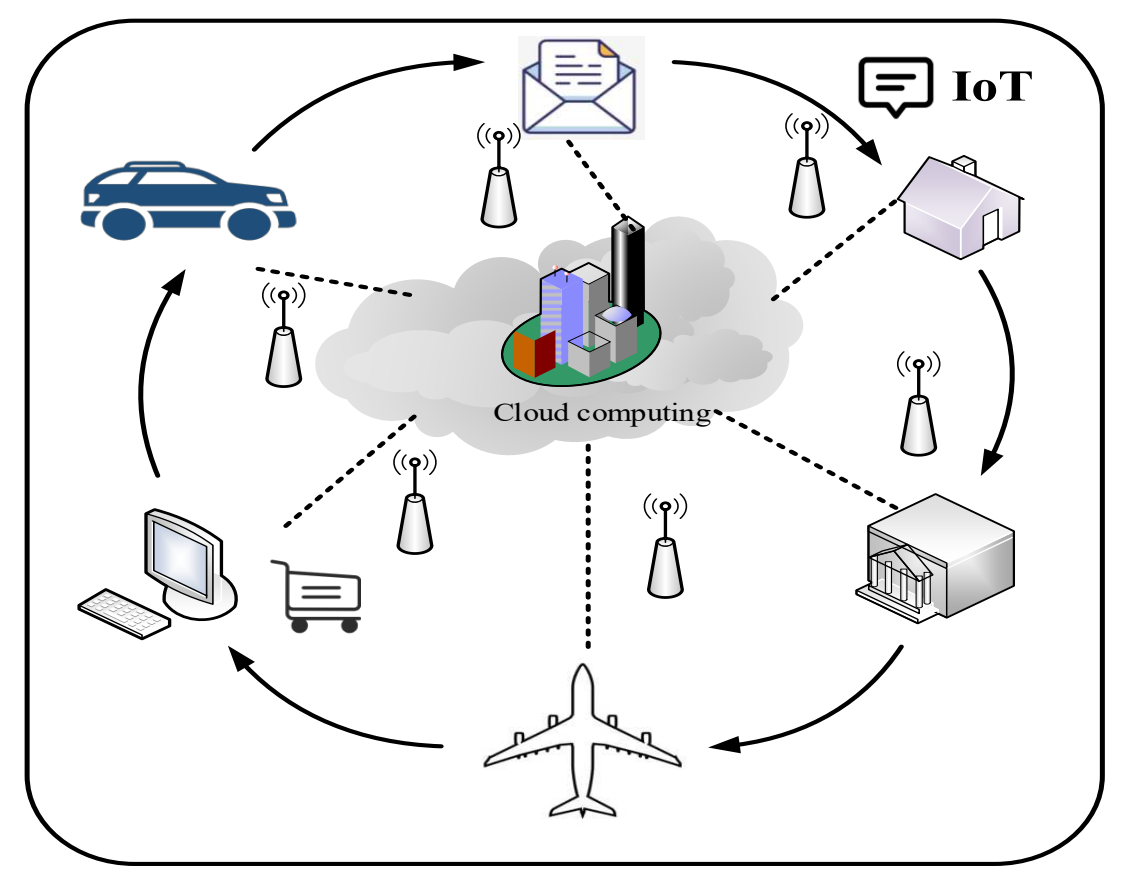

Figure 1. Smart tourism using the IoT.

The tourist experience is an important aspect of the business, and it has a direct impact on visitor satisfaction [8]. Traditional tourist tactics, on the other hand, may be unable to attract sufficient visitors to aid the economy's recovery. The phrase "smart tourism" was coined by the United Nations World Tourism Organization (UNWTO), which describes it as "green, ethical, clean and experience excellent tourism" [9]. Smart tourism is an important and forward-thinking strategy for transforming the tourism industry. The tourist industry has just lately begun to move toward more user-friendly intelligent systems based on "smart tourism" information technology [10]. Smart tourism is customercentric, which means it aims to meet all of a visitor's needs in terms of eating, housing, transportation, shopping, and entertainment. To put it another way, smart technology should be employed to enhance the visitor's experience at every stage of the journey.

Smart tourism strives to improve both passengers' tourist experiences and tourist destinations' competitiveness [11]. It will also contribute to the country's long-term development. Consumers may download smart applications to their phones to improve their city travel experience thanks to smart technology. Such smart apps can leverage local food, tales, customs, and so on to enrich the information provided to tourists. Visitors should be provided with relevant and helpful information based on individual data, behavior patterns, big data analysis, and other variables to aid them in making decisions prior to or during a trip. As a consequence, smart tourism is able to fulfill both immediate economic demands as well as long-term development objectives.

We propose a hybrid intelligence-based approach for predicting smart travel user behavior for the reasons indicated above. This is a hybrid intelligence-based technique to predicting KNN user behavior. According to cross-validation tests and performance evaluation methodologies, the system can categorize travel users' decision-making behaviors and has a good prediction ability. The results of the classification might be utilized to predict if the trip user will decide the destination in the near future.

The rest of this article is as follows. The Internet of Things' function and use in smart tourism are discussed in Section II. In Section III, the relevance of hybrid intelligence in addressing smart tourism 
problems is explored in depth. In Section IV, we'll look at a hybrid intelligence-based approach for anticipating KNN user behavior. This article comes to a close with Section V.

\section{Methods}

\subsection{Basics of Internet of Things}

The Internet of Things (IoT) is a flexible global network architecture that enables personality and interoperability based on current principles. Through the use of a huge number of devices, mobile phones, and RFID tags, the IoT is a critical focus of successful Internet growth. The arrangement of actuators and other equipment and through their large coverage of scenes and the ability to interact with each other, to achieve the ultimate goal, such as intelligent identification, monitoring, tracking, management and other important functions [12]. This means that in the Internet of Things, it is possible to build a scene that no longer needs human management and can realize information interaction. Concurrently, IoT also has other meanings. originally, the IoT is built on the network, and its heart remains the website. Secondly, as can be seen in Figure 2, any client can be integrated into the IoT and actualize data exchange, with the data processing. The Internet of Things with the three major distinctive features of information transmission and the gathering of data is considered a new generation of communication technology, and the most critical of these is information collection [13]. In short, the processing of data by the IoT is based on the massive collection of information.

In modern society, users esteem highly the comfort of their personal living environment. Through many sensors and actuators, the IoT can more effectively collect important things such as weather, temperature, humidity, location, historical records and different users' reactions to this. Information, which can configure the most personalized comfort ring for the user.

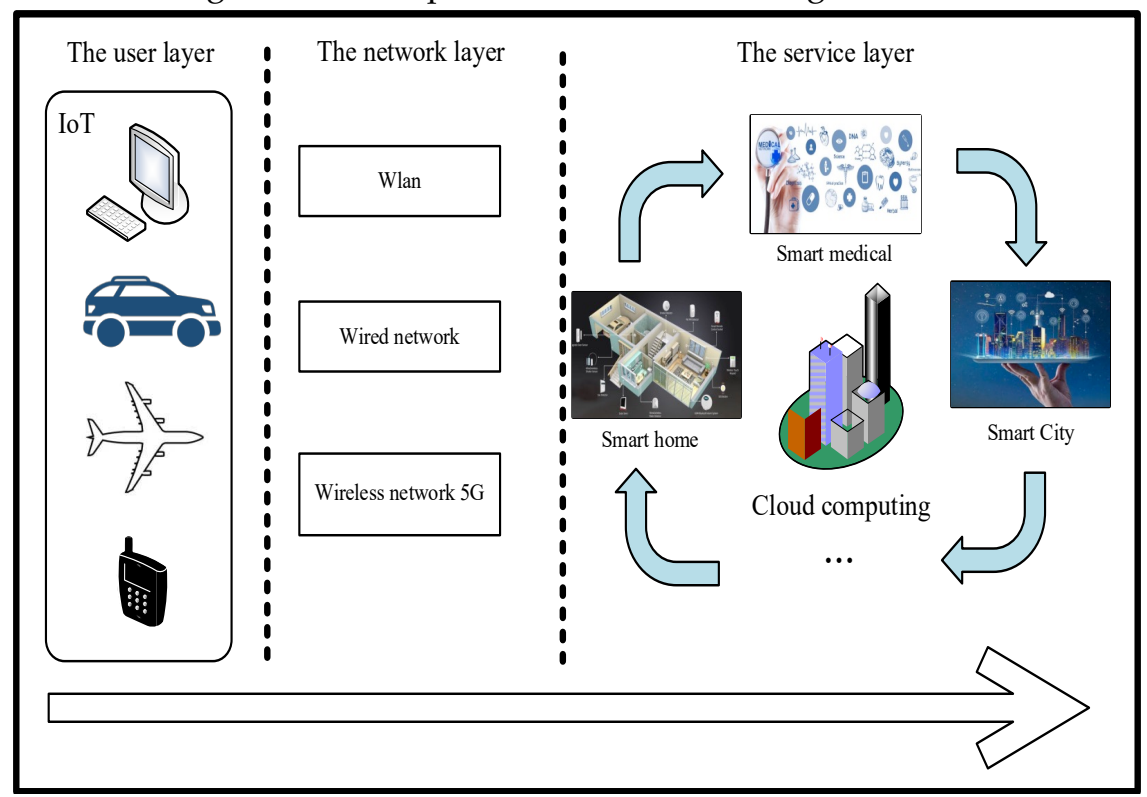

Figure 2. The 5G Internet of Things' design.

\subsection{G IoT for Smart Tourism}

The advancement of the third wave of industrialization will be accompanied by the continuous improvement of Internet information acquisition [15]. The IoT's growth will drive various fields, such as the economic field. With the advancement of the Internet of Things, we will also enter a new era of globalization. It will also drive the development of the tourism and entertainment industries. For example, the range and richness of perceptual information will Conducive to the intelligence of the tourism industry. And in smart tourism scenario is shown Figure 3, tourism services are convenient for people. The improvement of the IoT to the tourism industry can not only reduce carbon emissions, but also improve efficiency [16], and the improvement of scenic spot management systems can drive 
Tourism automation. The tourism scene built through the IoT can realize many functions. For the environment, it can realize gas detection, temperature and humidity monitoring, and animal and plant species monitoring. For the tourism industry itself, it can realize important functions such as tourism promotion, hotel and surrounding facilities services. Through the information collection of numerous sensors, numerous important data can be transmitted to the server, and safety monitoring and safety management are realized, and finally the purpose of statistics, analysis, and reporting is achieved.

Visitors can use VR, 5G, AI and other technologies to fully enjoy various beautiful scenery and culture without leaving home. For example: users' photos and videos can use technical methods to generate panoramic travel notes to record wonderful moments; and intelligent robots in scenic spots can quickly respond to the various needs of passengers with the help of $5 \mathrm{G}$ and voice recognition. In terms of safety, the invisible protective net in the scenic spot can be formed by the edge computing AI of the intelligent eagle eye system. To help tourists achieve better service and fine management, the scenic spot can also generate a panoramic experience that can be shared by tourists traveling.

Smart tourism has realized the integration of the Internet of Things and other applications in the four core technologies of mobile communication, cloud computing, the Internet of Things, and artificial intelligence, in order to maximize the Internet of Things' role in facilitating people's lives [17]. The scenario use of Internet of Things technology will become a critical component of smart tourism, meeting the requirements of an increasing number of consumers.

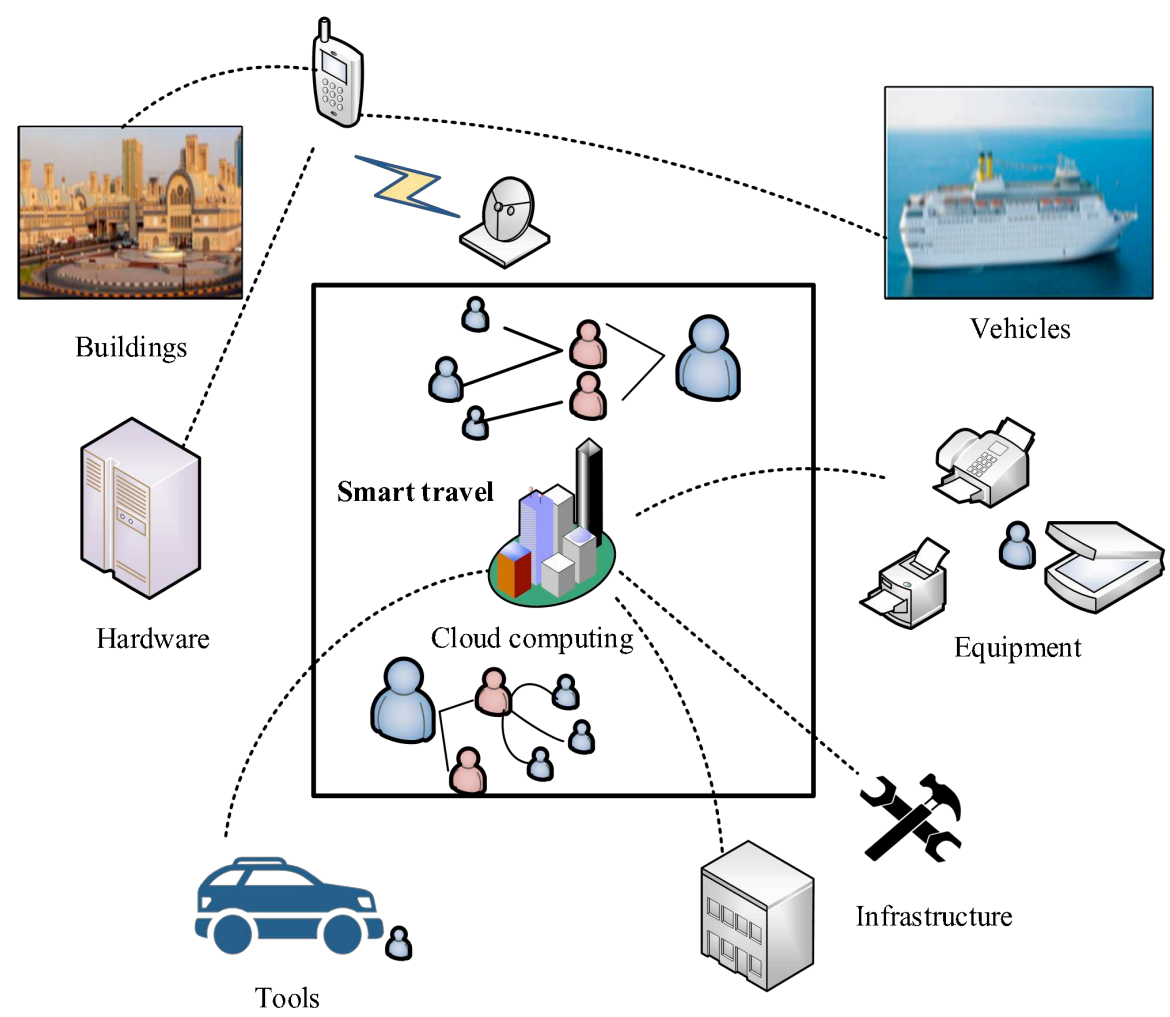

Figure 3. The implementation of intelligent tourism.

The implementation of the Internet of Things can also help passengers choose suitable destinations and arrange travel. For each user, smart travel services can provide personalized presentations, discover more ways to travel to the place, provide useful information services, and record travel experiences, comments and other information in a timely manner to further help passengers choose the best travel arrangements Land [18], improve travel efficiency.

Concurrently, the Internet of Things can also help tourists arrange their itineraries and improve the travel experience. Specifically, passengers can disclose public or private traffic data through their smartphones, which can improve the user's travel plan and further provide the latest information. At the same time, the system can automatically filter when the user interacts with the QR code and AR 
pictures on the mobile phone. Useless information and display effective information for travelers of different levels, which fully shows that the combination of tourism management system and the Internet of Things has changed the past travel methods [20].

\section{Hybrid Intelligence for Tourist Behavior Analysis}

\subsection{Basics Hybrid Intelligence}

Humanity and smart robots are now more inextricably linked. In our future world, humanmachine communication and collaboration will be essential [21]. On either hand, human problems are often complex, confusing, and open. Because humanity are the ultimate arbiters of the purpose of sentient devices, human participation in machines has remained consistent throughout their development [22]. Additionally, even if humans provide sufficient or limitless data resources for AI systems, it is hard to rule off the possibilities of human interference in the intelligent system. Many issues with AI systems must be solved, including how to recognize the differences and ambiguities of human language and comprehending of human communication systems, as well as how to reduce the dangers and even harms caused by AI limits. To overcome the limitations of existing artificial intelligence systems, the hybrid intelligence system combines machine and human intelligence [23].

Human intelligence may be incorporated into AI systems in hybrid intelligence systems, as illustrated in Figure 4, to complement machine functions throughout the life cycle. To address the limitations of AI systems, hybrid intelligence systems may share computational duties with humans as required. Human involvement may prevent AI systems from making mistakes and failing, and human input can lead to a virtuous development loop, enabling the system to learn constantly [24].

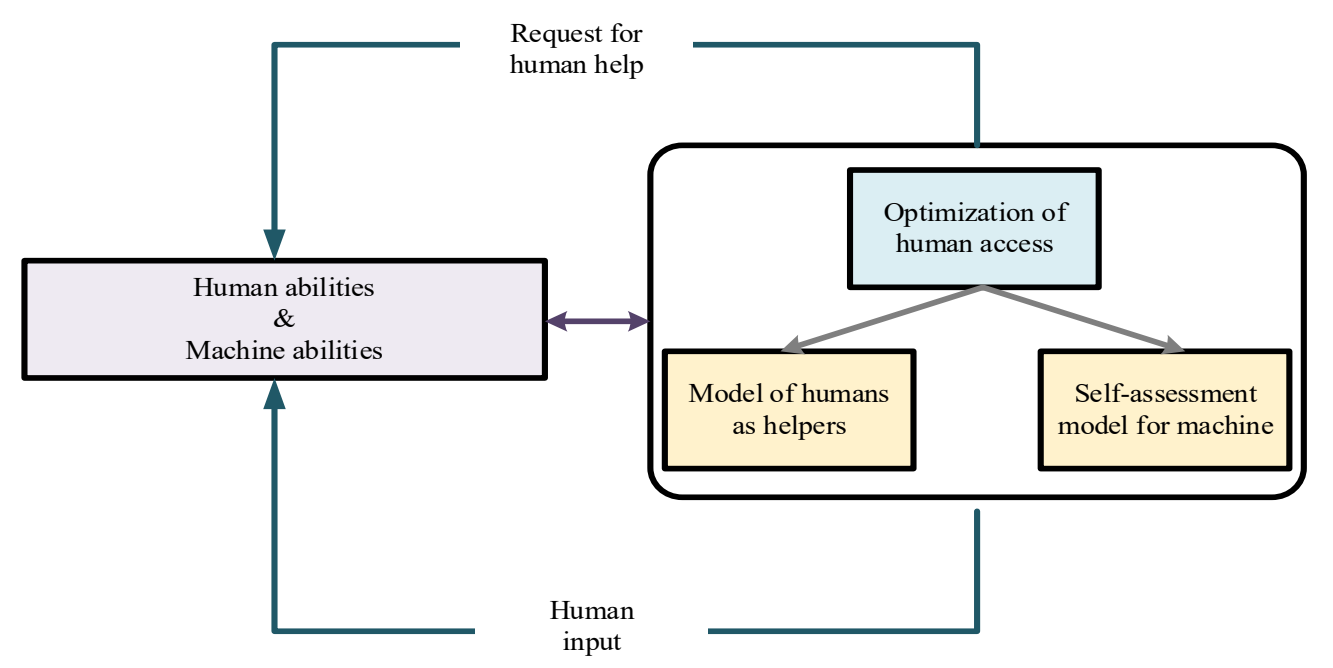

Figure 4. Reasoning capabilities for hybrid intelligence systems.

Additionally, hybrid intelligence systems may be used to address complex problems to train or classify using machine learning. In many fields, hybrid intelligence measures should be adopted differently. The basic framework of mixed intelligence systems, which should be seen as a mixed learning model, is shown in Figure 5. Hybrid intelligence models integrate artificial intelligence and human decision-making. It uses machine to learning (guided and unguided) to establish a model with training data or a small amount of samples then uses the model to evaluate fresh data [25]. The mix learning framework determines if the forecast should be manually adjusted or whether human participation is necessary when system is abnormal or computer predicted mistake rate is high and the system's information base is updated automatically. As a matter of fact, human involvement in the algorithm may increase the system's accurateness and reliability. Without doubt, in order to handle the majority of the job by computers, hybrid intelligence must reduce human participation. 
The hybrid learning model's intelligence may significantly enhance the scope and efficiency of activities that people can perform.

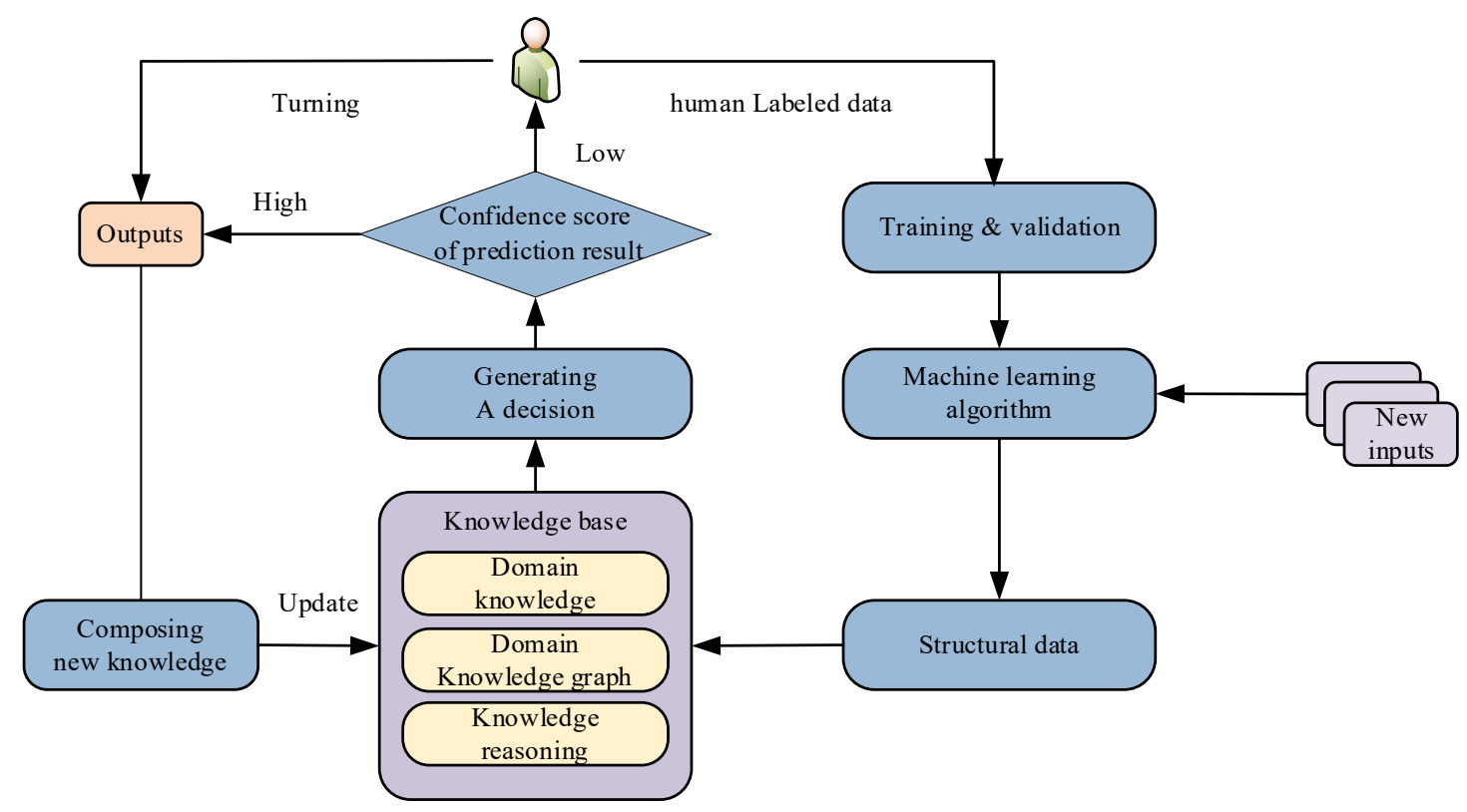

Figure 5. Basic framework of hybrid intelligence.

\subsection{Tourist Behaviors}

Many travel websites now offer the following services as a result of the development of different travel websites: travel information, traffic information, airline or hotel bookings, and so on. The ultimate destination will provide as a reminder to new tourist consumers of a reasonable journey time and a big amount of prior orders. We'd want to use current data to anticipate database managing information so that visitors may make decisions based on previous orders or browsing data, if we assess different indicators of user behavior.

\subsection{Hybrid Intelligence for Tourist Behavior Analysis}

We can assist customers in rapidly determining their travel destinations by studying their travel activities. For data analysis, hybrid intelligence is a useful method. We can organize and classify user behavior and anticipate user choice behaviors using artificial intelligence.

Unfortunately, using artificial intelligence methods to analyze consumers with limited private information or fewer prior orders would also provide inferior results and may even enhance the system's inaccuracy, which is why we use mixed intelligence approaches to analyze user behavior. Specific users may be deleted or tagged manually using manual techniques.

\section{Results and Discussion}

We use a large amount of travel data from more than 50,000 travel APP users to further evaluate the user's travel status. In order to ensure the rationality of the data, these data carefully include the user's order browsing record and the user's historical record on the APP Orders, the user's comments on the browsing history of different orders.

Through the comparative analysis of data, we believe that the user's choice of travel destination is predictable, and through the following characteristic variables, we can select the variable as the user's decision-making object and predict the decision-making result: 
1. The user searches for travel information before deciding to go to the destination

2. Estimate the value of travel destinations based on the history of user orders

3. Use a system that has been trained and predicted, combined with various indicators to determine whether the user will go to the destination

All of the above will ultimately ensure that the user's initial travel time is shortened

\subsection{Data Set Labeling}

Tag-based user positioning technology must be based on recording the user's ideal destination. At present, there are two main types of positioning technology: global positioning system (GPS) and location-based services (LBS) [26]. In the GPS method, the basic principle of a mobile station (MS) is to receive and measure the signal characteristics of at least four satellites from the 24 GPS satellites in the current network. This allows GPS to have an accurate system, and the user location based on GPS is extremely accurate. On the other hand, GPS-based methods make it necessary to place a GPS receiver in the mobile device, and this receiver will not only increase operating costs, but also Will consume more battery power, and more importantly, it will expose the user's geographic location to a certain extent.

LBS is another location-collecting technology. This technology collects the location of mobile terminal users through the wireless communication network of telecommunications and mobile operators [27]. This technology uses base stations to more accurately locate users' locations without the need for cellular the network coverage area uses blind spots. There is no requirement for the hardware configuration of the mobile terminal equipment, and it does not consume the power of the user's mobile terminal equipment. And compared with GPS, this technology is more practical. Therefore, the positioning method in this article is the LBS base station placement method. The location of the mobile user is measured by the signal received by the network base station. The base station monitors the signals and sends them to the central station for further processing and calculation to obtain the position of the mobile station [28], [29].

To estimate the MS position, the data fusion phase integrates measurement data from several BSs. Let $\left(\mathrm{x}_{\mathrm{m}}, \mathrm{ym}_{\mathrm{m}}\right)$ represent the MS position coordinates in the Cartesian coordinate system, as illustrated in Figure 6. Let the BSs'(BS1, $\mathrm{BS}_{2}$, and $\left.\mathrm{BS}_{3}\right)$ coordinates be $\left(\mathrm{x}_{1}, \mathrm{y}_{1}\right),\left(\mathrm{x}_{2}, \mathrm{y}_{2}\right)$, and $\left(\mathrm{x}_{3}, \mathrm{y}_{3}\right)$, respectively $(\mathrm{x} 3, \mathrm{y} 3)$. Only the $\mathrm{x}$ and $\mathrm{y}$ coordinates are used in the derivation for simplicity's sake, and the $\mathrm{z}$ coordinates are disregarded. This is the situation when BSs and mobile users are situated on a reasonably level surface.BS1 is set as the origin of the Cartesian coordinate system, that is, $(x 1, y 1)=$ $(0,0)$. The signal characteristics of the MS signal are angle and amplitude. To establish the user's approximate position, we utilize the time difference of arrival (TDOA) technique. For example, in Figure 6, the MSm detects through the BS that its coordinate position is within the coordinate range of the trip destination, and it is presumed that it has arrived at the destination in an acceptable period of time, and it may be recorded as "1"; If the MSm's coordinates do not match those of the attractions, it is presumed that it has not arrived at the destination in a timely way and may be logged as "0." 


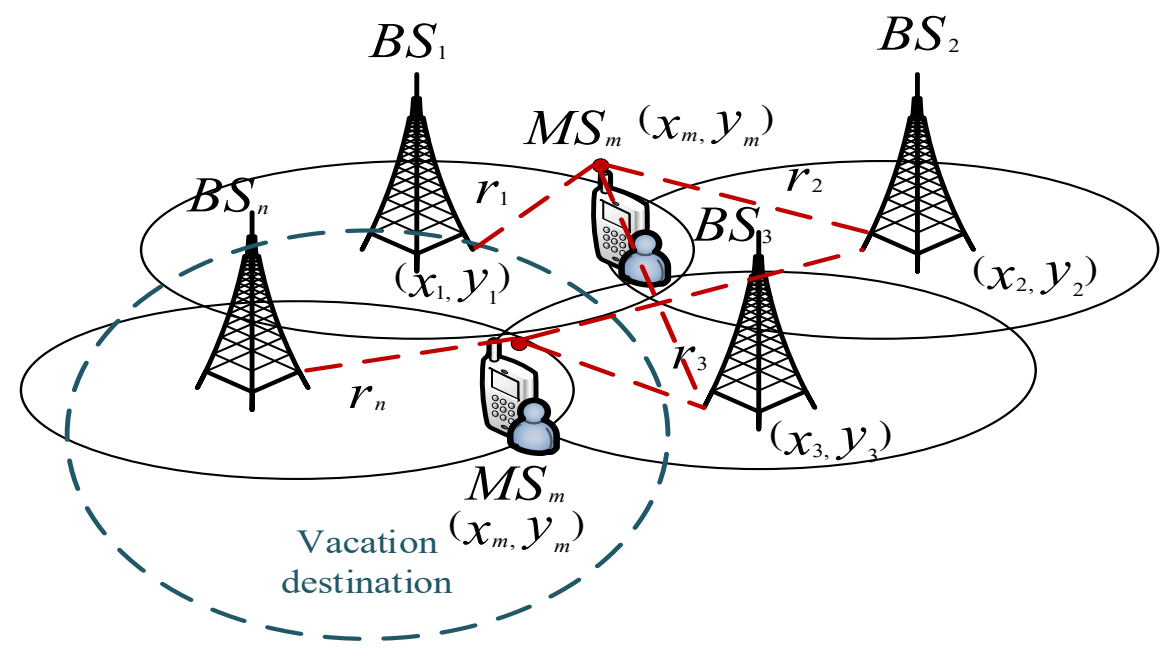

Figure 6. TDOA data fusion using multiple BSs

To better calculate the location of this point, we introduce the triangular dissection mechanism, which is an extension of the problem based on the intersection of two circles. To find the intersection of two circles, we need to determine whether the two circles intersect at point 1 , at point 2 , or at no intersection, and the solution is: introduce circles of radius $r$ and s with centers $(a, b)$ and $(c, d)$ through the following system of equations.

$$
\begin{aligned}
& (x-a)^{2}+(y-b)^{2}=r^{2} \\
& (x-c)^{2}+(y-d)^{2}=s^{2}
\end{aligned}
$$

In order to solve the equations, we can convert these equations to linear form. One of the solutions is to find the intersection point by translation and rotation of the axes. The algorithm is discussed as follows: The intersection points of two circles $(x 1, y 1)$ and $(x 2, y 2)$ are calculated as follows.

$$
\begin{gathered}
\mathrm{e}=\mathrm{c}-\mathrm{a} \\
\mathrm{f}=\mathrm{d}-\mathrm{b} \\
p=\sqrt{e^{2}+f^{2}} \\
k=\frac{p^{2}+r^{2}-s^{2}}{2 p}
\end{gathered}
$$

So we get the result:

$$
\begin{aligned}
& x_{1}=a+\frac{\mathrm{e} k}{p}+\frac{f}{p} * \sqrt{r^{2}-k^{2}} \\
& y_{1}=b+\frac{f k}{p}+\frac{e}{p} * \sqrt{r^{2}-k^{2}} \\
& x_{2}=a+\frac{\mathrm{e} k}{p}-\frac{f}{p} * \sqrt{r^{2}-k^{2}} \\
& y_{2}=b+\frac{f k}{p}-\frac{e}{p} * \sqrt{r^{2}-k^{2}}
\end{aligned}
$$

So we get the extended solution of this problem and find the point involving the intersection of the three circles called intersection and the most suitable point

\subsection{KNN Classification for Tourist Behavior}

After that, we need to classify user behavior data, the classification method used is the k-nearest neighbor (KNN) technique. KNN is a distance-based supervised learning method. This method uses the nearest neighbor category to identify the new data category [33]. This method separates different 
new data by calculating the distance between the new data and the original marked data point [32]. When calculating the distance, we use the Euclidean technique, and finally get the closest training data of the first $k$ distances. See who has the most data in each category, and use this category as the new data category. When it comes to selecting $\mathrm{K}$ values, there is no one-size-fits-all approach. A smaller number is usually chosen depending on the sample distribution, and an appropriate $\mathrm{K}$ value may be found via cross-validation.

\subsection{KNN Tourist Activity Classification based on HITL}

The classic KNN algorithm without the involvement of artificial intelligence suffer from inaccuracies in processing some more complicated data's edge information. The introduction of incorporating embedding human intelligence into intelligent systems, enhanced cognitive processes for the aim of analysis and reaction in a hazy and unclear situation issues may be closely coupled with machine intelligent systems. As a result, the two adapting to one another and cooperating to create a two-way information and control structure. Bringing human vision together, ability to think, ability to interpret information in a machine, and storage ability can result in "1 $+1>2$ " boosted intelligence hybridization. The KNN algorithm is then used in conjunction with hybrid intelligence to classify consumers.

In order to observe the classifications error rate of $\mathrm{KNN}$, we define the training error rate as the ratio of KNN training set tags to input tags, and the error rate is defined as:

$$
\frac{1}{\mathrm{~K} \sum_{x_{i \in N_{\mathrm{K}}(x)}} I\left(y_{i} \neq c_{i}\right)}=1-1 / \mathrm{K} \sum_{x_{i} \in N_{\mathrm{K}}(x)} I\left(y_{i}=c_{j}\right)
$$

As a result, appropriate the value of $\mathrm{k}$ can be increased.

$$
1 / \mathrm{K} \sum_{x_{i} \in N_{\mathrm{K}}(x)} I\left(y_{i}=c_{j}\right)
$$

in the exercise set

To test the correctness of the categorization, we utilize to get the best $\mathrm{K}$ value, use grid search and cross-validation [34]. We began by screening the data. There were a total of 40307 data after filtering. The information was split into six categories. Cross-validation was performed on the first five groups. There were 6717 data points in each category. out of a total of 33585, with the sixth group having 6722 data. For the last accuracy check, the data is used.

For training, we use four sets of data and one set of data for testing. for testing in cross-validation. Each data set has a chance to become the test set, as illustrated. When $\mathrm{K}$ is equal to 8, the training error rate is equal to low, according to the grid search results. Figure 7 depicts the accuracy of the training and test sets with varying numbers of neighbors. Finally, the final verification set of 6722 data was reviewed, and the accurate rate was 84.06 percent. 


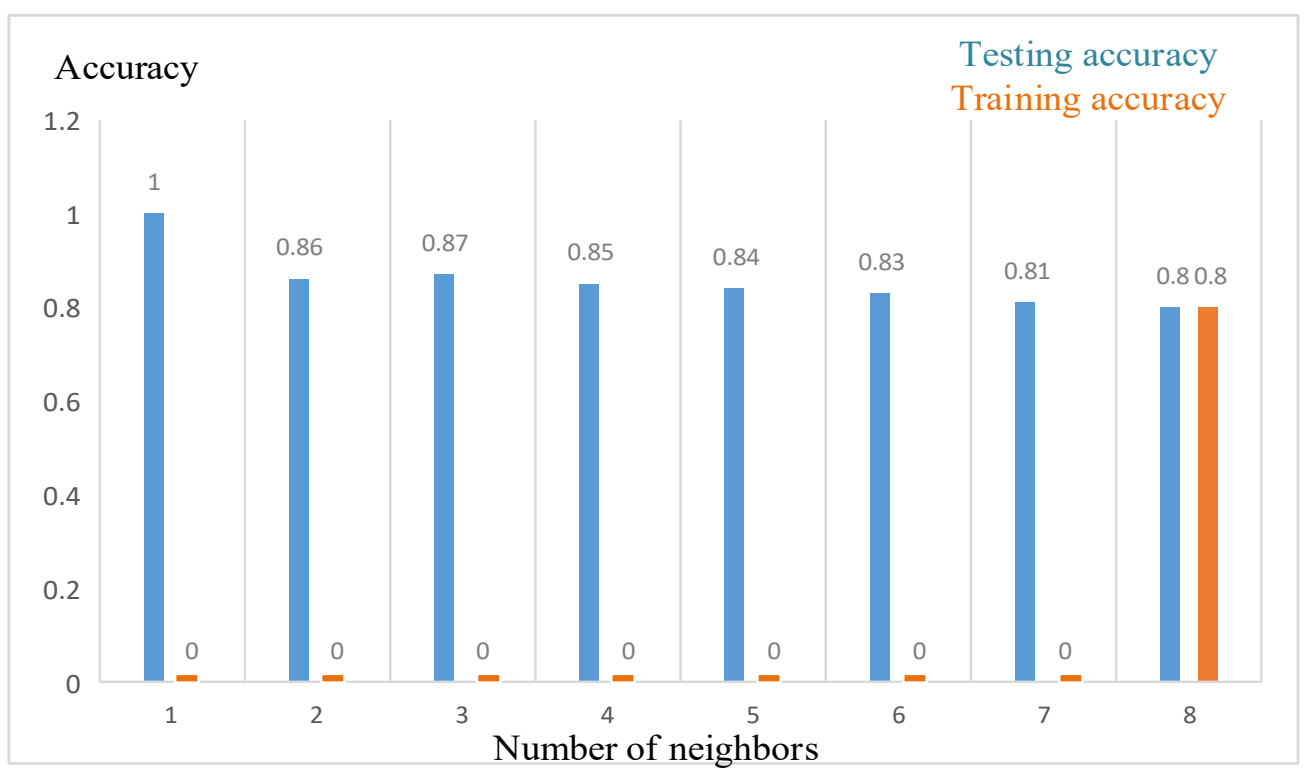

Figure 7. The cross-validation rate is accurate.

In the realm of machine learning, a confusion matrix is a specific matrix used to illustrate the performance of an algorithm [35]. In predictive analysis, the confusion matrix is a table that comprises false positives, false positives, genuine positives, and true positives. It's simple to check if the learning machine obfuscates two classes that are similar using an obfuscation matrix [36]. The confusion matrix is used to visualize the performance of an algorithm [29]. In predictive analysis, the confusion matrix is a table made up of False Positives (FP), False Negatives (FN), True Positives (TP), and True Negatives (TN). Obviously, assuming that TP, FP, TN, and FN reflect the relevant sample numbers:

$$
\mathrm{TP}+\mathrm{FP}+\mathrm{TN}+\mathrm{FN}=\text { Total }
$$

Table 1 depicts the "Confusion matrix" of this experiment's classification conclusions.

\begin{tabular}{|c|c|c|}
\hline True label & Confusion matrix \\
$\begin{array}{c}\text { Predicted } \\
\text { label }\end{array}$ & 0 & 1 \\
\hline 0 & 13289 & 2542 \\
\hline 1 & 184 & 108 \\
\hline
\end{tabular}

Table 1. The confusion matrix.

The ROC curve may be used to investigate the features of the model's distinguishing types in order to assess the model's generalization performance more fully [37]. The ROC curve is usually more effective when the class distribution is not uniform. The "True Positive Rate" is defined as (14) on the vertical axis and "False Positive Rate" is defined as (14) on the horizontal axis of the ROC curve (15).

$$
\begin{aligned}
& \mathrm{TPR}=\frac{\mathrm{TP}}{\mathrm{TP}+\mathrm{FN}} \\
& \mathrm{FPR}=\frac{\mathrm{FP}}{\mathrm{TN}+\mathrm{FP}}
\end{aligned}
$$

The region beneath the ROC curve (AUC) is a metric used to assess the learning machine's performance [38]. AUC is calculated by adding the area of each component under the ROC curve. The AUC may be calculated by linking a sequence of coordinate points $(x 1, y 1),(x 2, y 2),(x 3, y 3)$, etc., 
(xm, ym) a sequential manner to construct the ROC curve.

$$
\mathrm{AUC}=\frac{1}{2} \sum_{i=1}^{m-1}\left(x_{i+1}-x_{i}\right) \bullet\left(y_{i+1}+y_{i}\right)
$$

The difference reflected in the AUC-ROC curve is due to the different performance statistics caused by the different threshold settings. ROC is a probability curve, and AUC is a measure of separability. ROC symbolizes the ability of characteristic values to stand out from other data. The larger the AUC, the better the model can distinguish between 0 and 1 , so it can better predict the user's decision.

The ROC curve is displayed using TPR and FPR. As shown in Figure 8, FPR is plotted on the xaxis and TPR is plotted on the $y$-axis. The AUC of the different classification results of the three different parameters are represented by regions $\mathrm{A}, \mathrm{B}$, and $\mathrm{C}$, respectively. Through the comparative analysis of the three curves, we can get the conclusion that the AUC-ROC curve corresponding to AUC-A has the best performance.

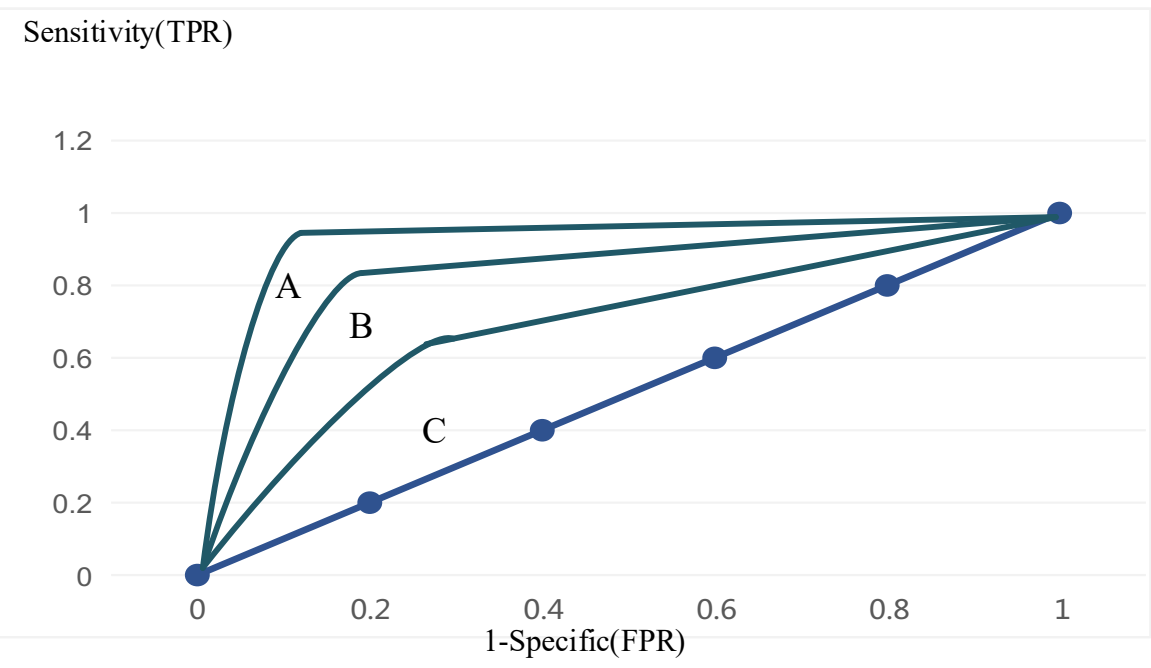

Figure 8. Areas under different ROC-AUC curves compared.

Figure 9 displays the ROC curve for the classification model we developed. We get a semiellipse approximately $\mathrm{AUC}=0.809$, which shows that our model is very capable of classification As a result of this model, we can classify whether or not travelers pick destination A. Users are supplied with relevant services based on the categorization findings. 


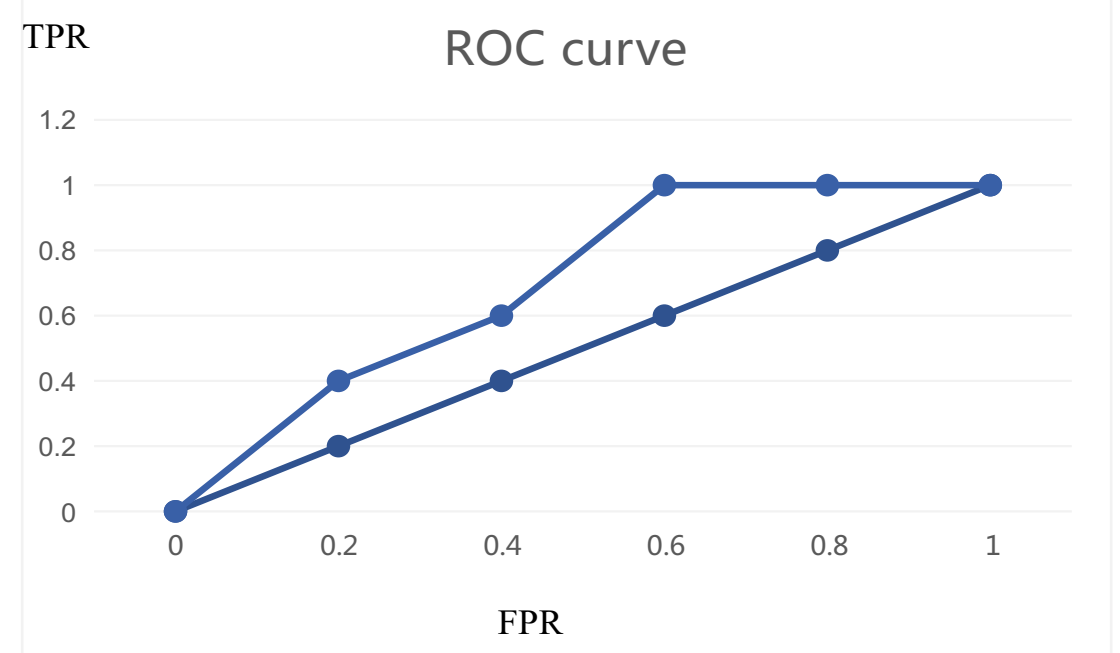

Figure 9. The provided model's ROC curve.

\section{Conclusions}

As tourism grows, incorporating the IoT into tourist projects is a very promising technology. Smart tourism makes use of the Internet of Things to improve data analysis and integration, allowing for quick and easy information transmission between users. To put it another way, the Internet of Things will become a critical component in meeting the demands of an entirely new generation of visitors. As a result, we present a hybrid intelligence KNN classification-based tourist behavior decision-making approach. Based on previous order information and browsing history, this categorization system may effectively assist travelers in deciding whether or not to visit a specific vacation location. The method's efficacy is demonstrated by the results of cross-validation studies and performance evaluation.

Availability of data and materials: Data is private data is not public.

Conflicts of Interest: There are no conflicts of interest declared by the authors.

Founding: This work was supported by Xi'an University of Architecture and Technology.

Authors Contribution: WZ participated in the design of the study and performed the statistical analysis. HC conceived of the study, and participated in its design and coordination and helped to draft the manuscript. All authors read and approved the final manuscript.

List of Abbreviations

\begin{tabular}{|c|c|}
\hline IoT & Internet of Things \\
\hline UNWTO & United nations world tourism organization \\
\hline GPS & Global positioning system \\
\hline LBS & Location-based services \\
\hline TDOA & Time difference of arrival \\
\hline KNN & k-nearest neighbor \\
\hline FP & False Positives \\
\hline FN & False Negatives \\
\hline TP & True Positives \\
\hline
\end{tabular}




\begin{tabular}{|c|c|}
\hline TN & True Negatives \\
\hline
\end{tabular}

\section{References}

1. N. Dlodlo, O. Gcaba and A. Smith, "Internet of things technologies in smart cities," 2016 IST-Africa Week Conference, 2016, pp. 1-7.

2. C. Schmitt, C. Anliker and B. Stiller, "Pull support for IoT applications using mobile access framework WebMaDa," 2016 IEEE 3rd World Forum on Internet of Things (WF-IoT), 2016, pp. 377-382

3. S. Rhee, "Catalyzing the Internet of Things and smart cities: Global City Teams Challenge," 2016 1st International Workshop on Science of Smart City Operations and Platforms Engineering (SCOPE) in partnership with Global City Teams Challenge (GCTC) (SCOPE - GCTC), 2016, pp. 1-4

4. E. Sohn, K. Noh, B. Lee and O. Kwon, "Bibliometric Network Analysis and Visualization of Research and Development Trends in Precision Medicine," 2018 IEEE/ACM International Conference on Advances in Social Networks Analysis and Mining (ASONAM), 2018, pp. 727-730.

5. A. N. Kazak and N. Shamayeva, "Separate Aspects of Smart Cities Security," 2018 IEEE International Conference "Quality Management, Transport and Information Security, Information Technologies" (IT\&QM\&IS), 2018, pp. 216-218.

6. R. Nurbadi, A. Amyus, B. Pratama, A. F. Kanz and A. N. Fajar, "Smart Travel System Based on Service Oriented Architecture," 2018 Indonesian Association for Pattern Recognition International Conference (INAPR), 2018, pp. 256-261.

7. C. Xu, X. Huang, J. Zhu and K. Zhang, "Research on the Construction of Sanya Smart Tourism City Based on Internet and Big Data," 2018 International Conference on Intelligent Transportation, Big Data \& Smart City (ICITBS), 2018, pp. 125-128

8. B. P. Gautam, H. Asami, A. Batajoo and T. Fujisaki, "Regional Revival through IoT Enabled Smart Tourism Process Framework (STPF): A Proposal," 2016 Joint 8th International Conference on Soft Computing and Intelligent Systems (SCIS) and 17th International Symposium on Advanced Intelligent Systems (ISIS), 2016, pp. 743-748.

9. E. Balandina, S. Balandin, Y. Koucheryavy, S. Balandin and D. Mouromtsev, "Innovative e-Tourism Services on Top of Geo2Tag LBS Platform," 2015 11th International Conference on Signal-Image Technology \& Internet-Based Systems (SITIS), 2015, pp. 752-759.

10. E. Balandina, S. Balandin, Y. Koucheryavy and D. Mouromtsev, "IoT Use Cases in Healthcare and Tourism," 2015 IEEE 17th Conference on Business Informatics, 2015, pp. 37-44.

11. J. C. Hung, V. Hsu and Y. Wang, "A Smart-Travel System Based on Social Network Service for Cloud Environment," 2011 Third International Conference on Intelligent Networking and Collaborative Systems, 2011, pp. 514-519.

12. S. Muthuraman and M. Al Haziazi, "Smart Tourism Destination - New Exploration towards Sustainable Development in Sultanate of Oman," 2019 5th International Conference on Information Management (ICIM), 2019, pp. 332-335.

13. O. Gcaba and N. Dlodlo, "The internet of things for South African tourism," 2016 IST-Africa Week Conference, 2016, pp. 1-8.

14. S. Ziegler, "Considerations on IPv6 scalability for the Internet of Things - Towards an intergalactic Internet," 2017 Global Internet of Things Summit (GIoTS), 2017, pp. 1-4.

15. S. Vashi, J. Ram, J. Modi, S. Verma and C. Prakash, "Internet of Things (IoT): A vision, architectural elements, and security issues," 2017 International Conference on I-SMAC (IoT in Social, Mobile, Analytics and Cloud) (I-SMAC), 2017, pp. 492-496.

16. W. Guo, N. Zhao and H. Shao, "IT Investment Efficiency Analysis of Equipment Manufacturing Industry Based on Two-stage Nonparametric Model," 2010 International Conference on Challenges in Environmental Science and Computer Engineering, 2010, pp. 21-24.

17. C. Lee, T. Ding and Z. H. Lin, "Integration of ERP and Internet of Things in Intelligent Enterprise Management," 2018 1st International Cognitive Cities Conference (IC3), 2018, pp. 246-247.

18. A. A. Emhmed and K. Chellapan, "GIS-based mobile tourism architecture prototype for Libya (A case study)," 2010 International Symposium on Information Technology, 2010, pp. 1-3. 
19. Y. Lin, "The Application of the Internet of Things in Hainan Tourism Scenic Spot," 2011 Seventh International Conference on Computational Intelligence and Security, 2011, pp. 1549-1553.

20. L. Tao, Y. Dong, L. Qi and S. Lv, "Fuzzy TOPSIS Approach Based on Axiomatic Fuzzy Set for Assessing Thermal-Energy Storage in Concentrated Solar Power (CSP) Systems," 2017 International Conference on Network and Information Systems for Computers (ICNISC), 2017, pp. 158-162.

21. X. Jin, T. Xu, F. Zhou and Y. Wu, "Technical Direction Analysis on Multi-Branch Large-Sample Wargaming: A Hybrid Intelligence Based Solution to Future Command \& Control," 2019 11th International Conference on Intelligent Human-Machine Systems and Cybernetics (IHMSC), 2019, pp. 322-325.

22. L. Li and M. Cui, "Human-system cooperative hybrid augmented intelligence based dynamic dispatching framework of semiconductor wafer fabrication facility," 2018 IEEE International Conference on Smart Manufacturing, Industrial \& Logistics Engineering (SMILE), 2018, pp. 1-5.

23. H. Liang, L. Yang, H. Cheng, W. Tu and M. Xu, "Human-in-the-loop reinforcement learning," 2017 Chinese Automation Congress (CAC), 2017, pp. 4511-4518.

24. A. Freedy et al., "Multiagent Adjustable Autonomy Framework (MAAF) for multi-robot, multi-human teams," 2008 International Symposium on Collaborative Technologies and Systems, 2008, pp. 498-505.

25. D. Wu, R. Bao, Z. Li, H. Wang, H. Zhang and R. Wang, "Edge-Cloud Collaboration Enabled Video Service Enhancement: A Hybrid Human-Artificial Intelligence Scheme," in IEEE Transactions on Multimedia.

26. M. Tahmassebpour, "Detecting fault location in wireless sensor networks using an exploratory method based on fuzzy logic," 2017 IEEE 4th International Conference on Knowledge-Based Engineering and Innovation (KBEI), 2017, pp. 0465-0469.

27. X. Li, G. Sun and X. Wang, "Mobile Positioning System Based on the Wireless Sensor Network in Buildings," 2009 5th International Conference on Wireless Communications, Networking and Mobile Computing, 2009, pp. 1-4.

28. M. R. Regitha, N. Antony, S. Sivan and V. Paul, "Optimization of handoff delay and location prediction of mobile station using GPS integrated SIM module in cellular networks," 2017 International Conference on Intelligent Computing, Instrumentation and Control Technologies (ICICICT), 2017, pp. 582-587.

29. A. J. Weiss, "On the accuracy of a cellular location system based on RSS measurements," in IEEE Transactions on Vehicular Technology, vol. 52, no. 6, pp. 1508-1518, Nov. 2003.

30. Haifeng Li, Jingtao Zhen, Yan Zhang and Lei Chen, "Design of medical wireless location network based on Zigbee protocol," 2007 IET Conference on Wireless, Mobile and Sensor Networks (CCWMSN07), 2007, pp. 709-712.

31. Y. T. Chan and K. C. Ho, "A simple and efficient estimator for hyperbolic location," in IEEE Transactions on Signal Processing, vol. 42, no. 8, pp. 1905-1915, Aug. 1994.

32. Y. T. Quek, W. L. Woo and T. Logenthiran, "DC equipment identification using K-means clustering and kNN classification techniques," 2016 IEEE Region 10 Conference (TENCON), 2016, pp. 777-780.

33. Lijuan Zhou, Linshuang Wang, Xuebin Ge and Qian Shi, "A clustering-Based KNN improved algorithm CLKNN for text classification," 2010 2nd International Asia Conference on Informatics in Control, Automation and Robotics (CAR 2010), 2010, pp. 212-215.

34. S. Sun and R. Huang, "An adaptive k-nearest neighbor algorithm," 2010 Seventh International Conference on Fuzzy Systems and Knowledge Discovery, 2010, pp. 91-94.

35. X. Zhang, Bicheng Li and Xianzhu Sun, "A k-nearest neighbor text classification algorithm based on fuzzy integral," 2010 Sixth International Conference on Natural Computation, 2010, pp. 2228-2231.

36. X. Zhang, Bicheng Li and Xianzhu Sun, "A k-nearest neighbor text classification algorithm based on fuzzy integral," 2010 Sixth International Conference on Natural Computation, 2010, pp. 2228-2231.

37. E. Keedwell, "An analysis of the area under the ROC curve and its use as a metric for comparing clinical scorecards," 2014 IEEE International Conference on Bioinformatics and Biomedicine (BIBM), 2014, pp. 2429.

38. A. Slaby, "ROC Analysis with Matlab," 2007 29th International Conference on Information Technology Interfaces, 2007, pp. 191-196. 


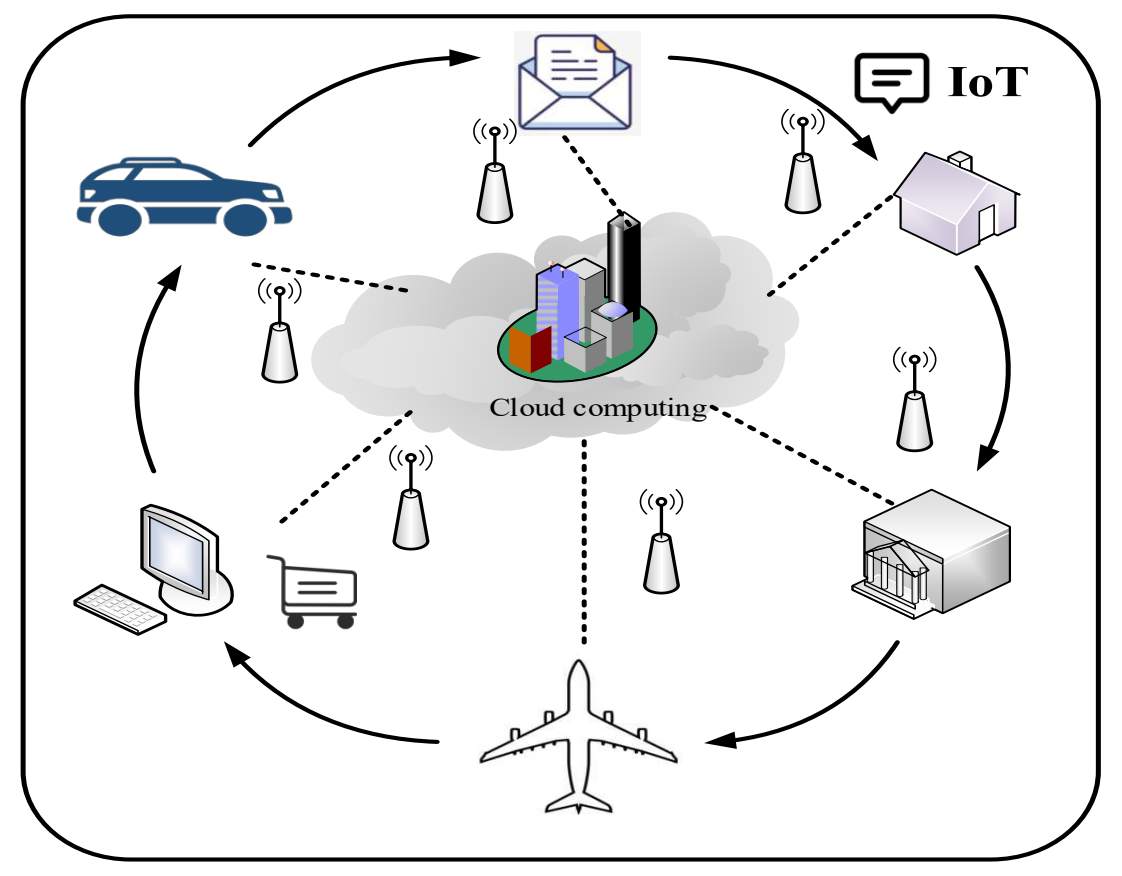

Figure 1. Smart tourism using the IoT.

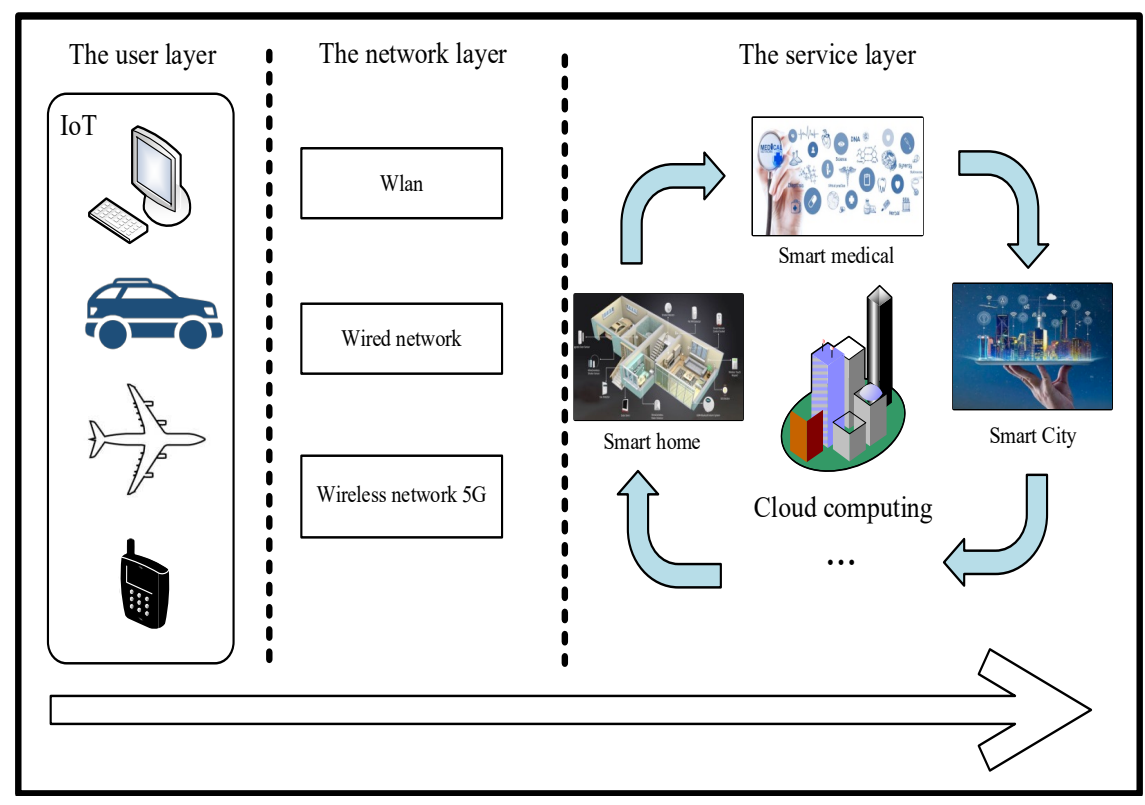

Figure 2. The 5G Internet of Things' design. 


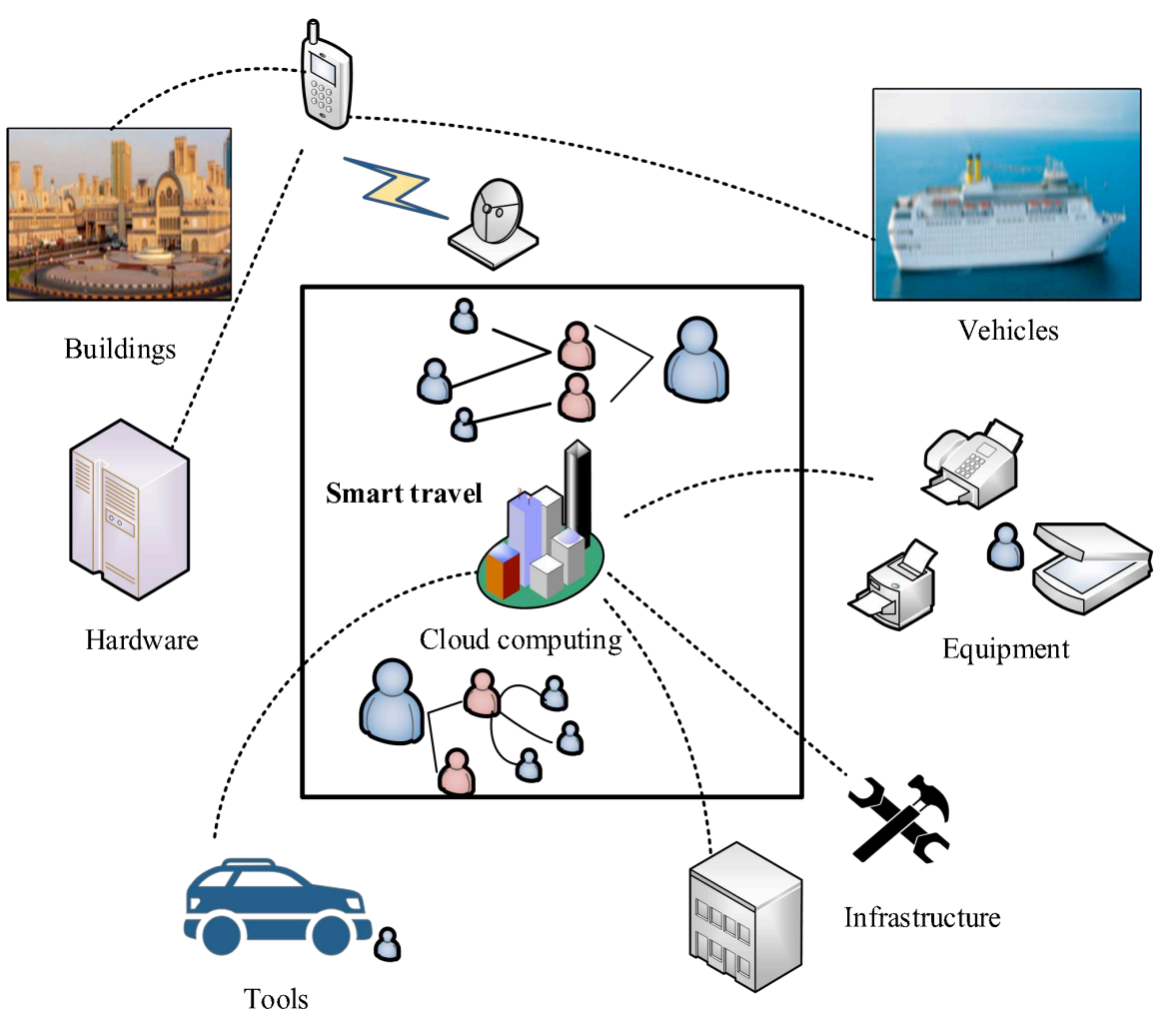

Figure 3. The implementation of intelligent tourism.

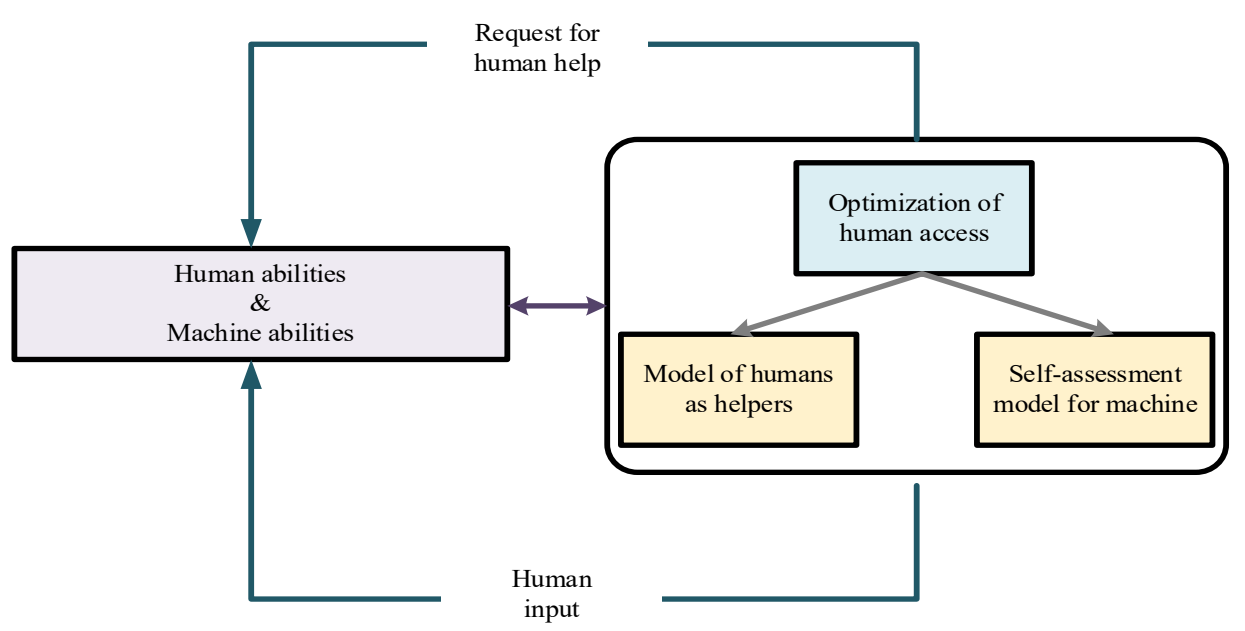

Figure 4. Reasoning capabilities for hybrid intelligence systems. 


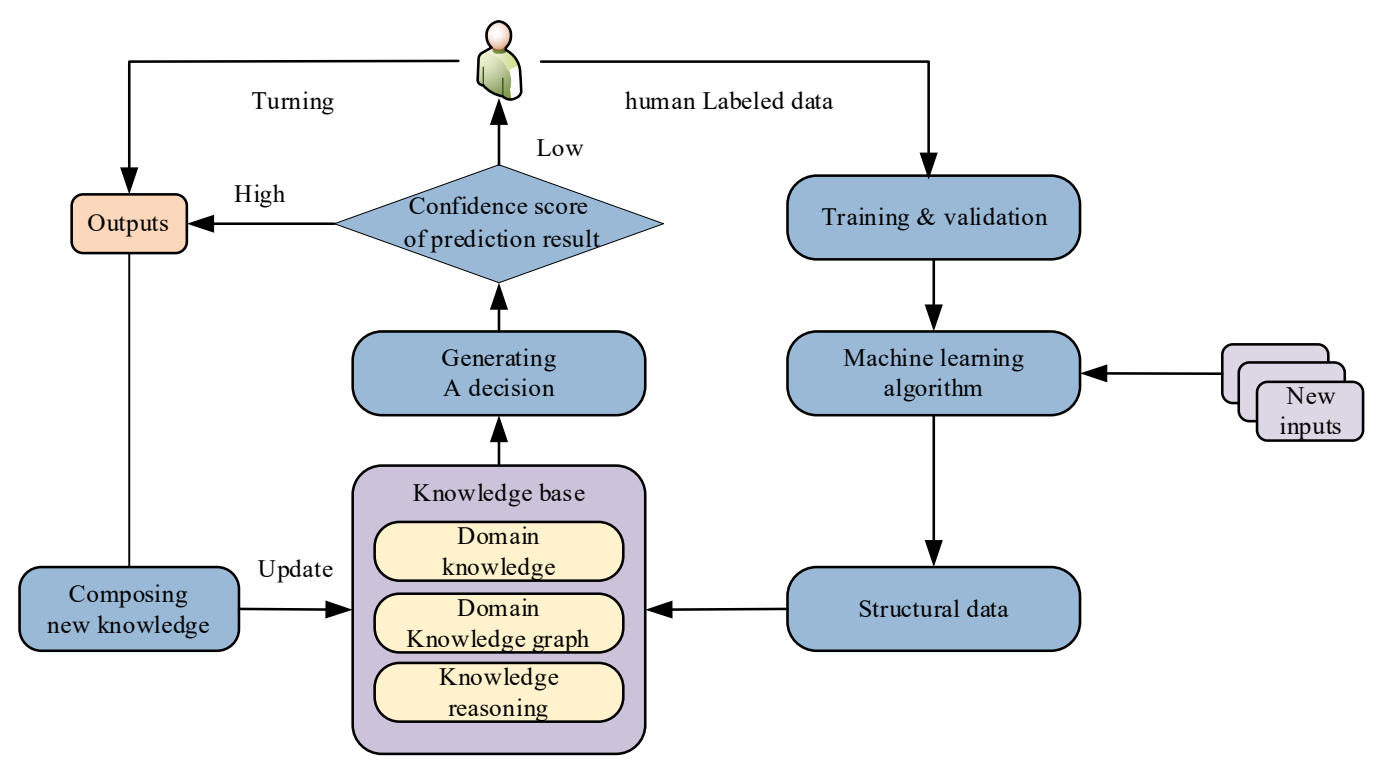

Figure 5. Basic framework of hybrid intelligence.

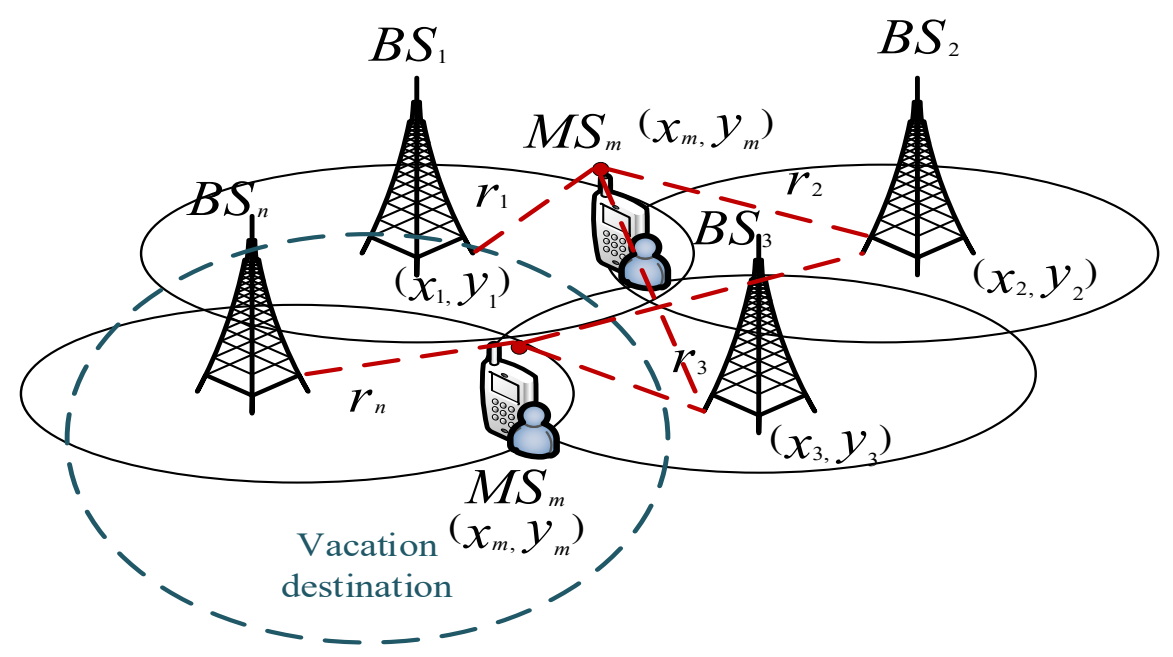

Figure 6. TDOA data fusion using multiple BSs

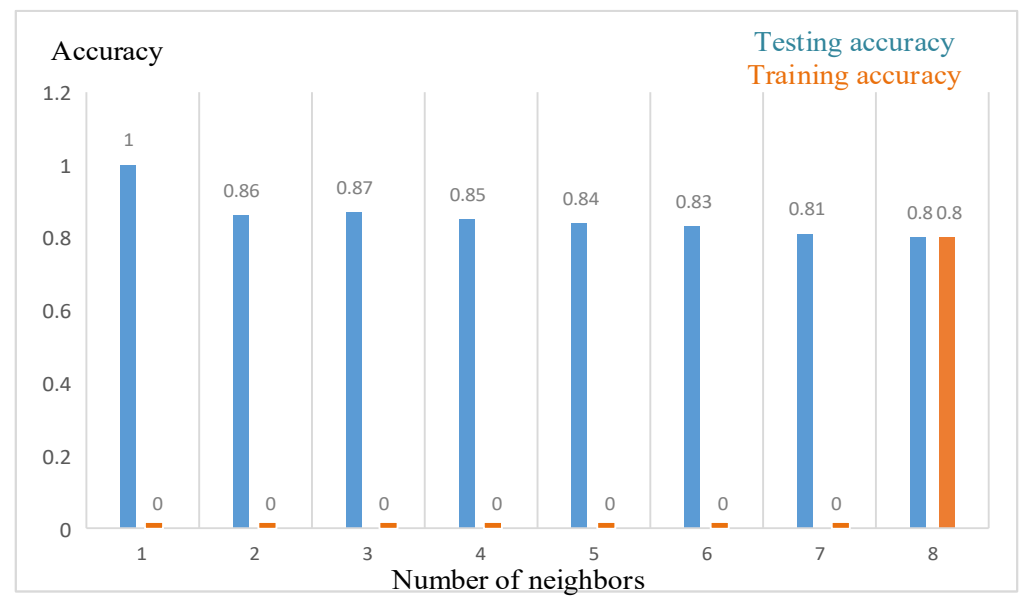

Figure 7. The cross-validation rate is accurate. 
Sensitivity(TPR)

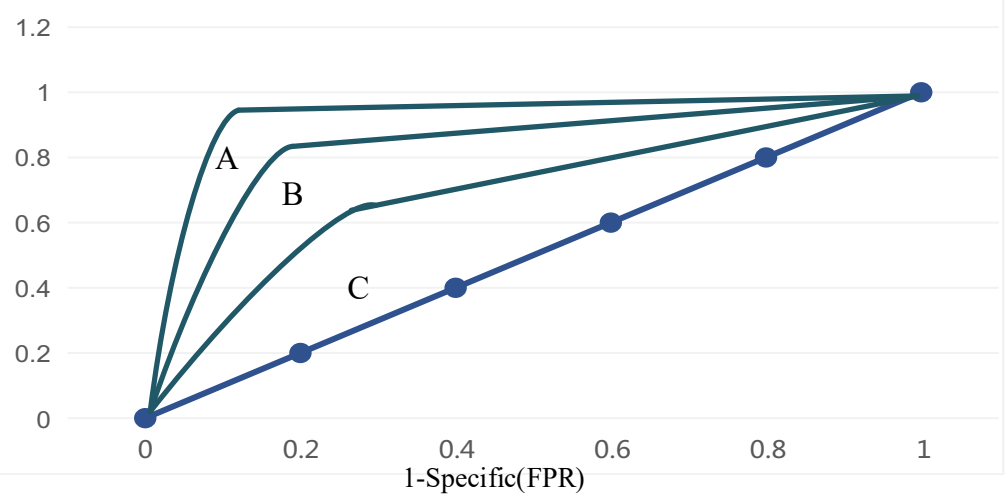

Figure 8. Areas under different ROC-AUC curves compared.

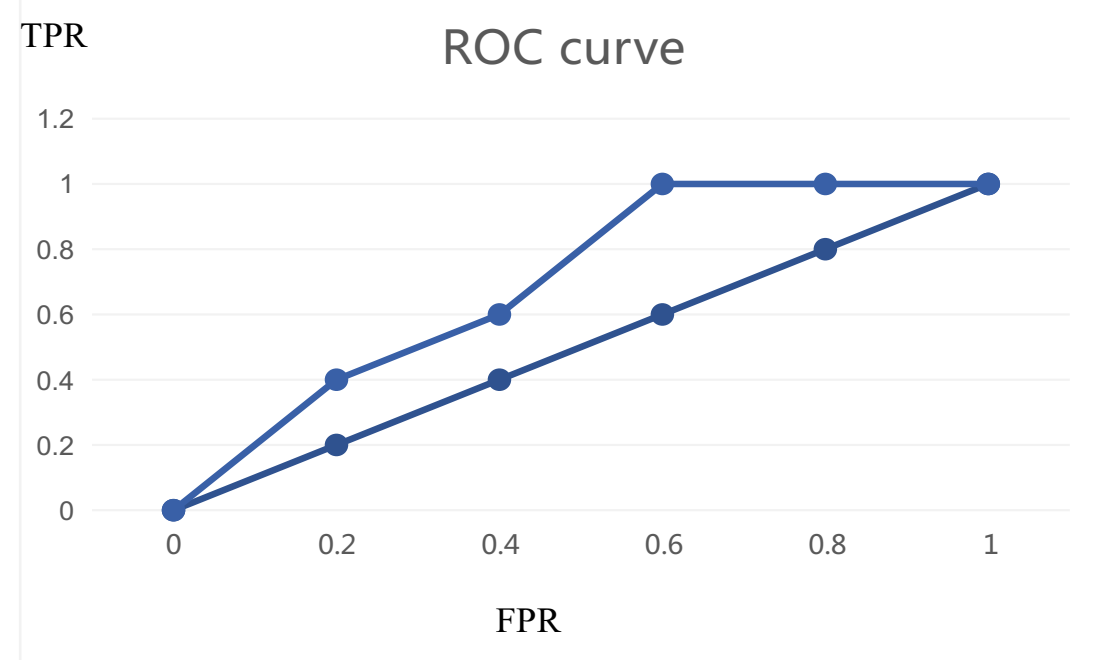

Figure 9. The provided model's ROC curve. 